
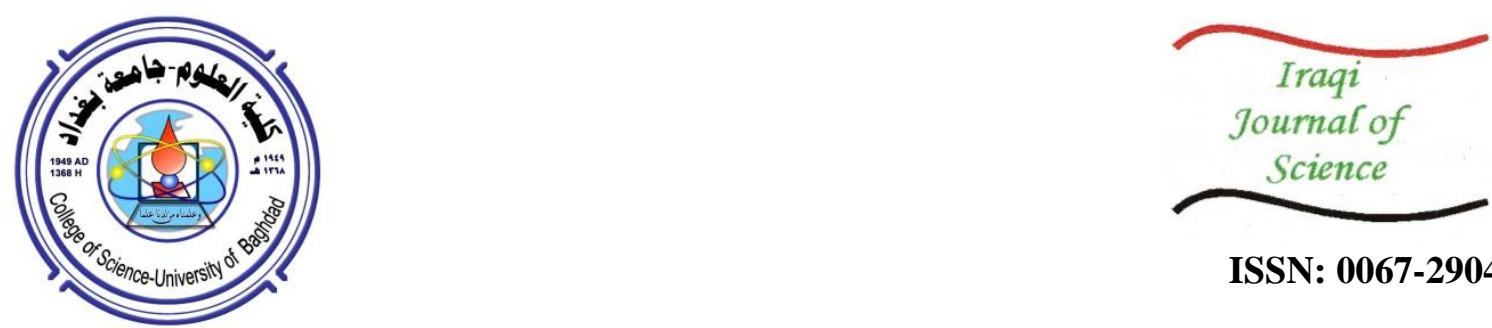

ISSN: 0067-2904

\title{
Oscillatory Flow MHD of Jeffrey Fluid with Temperature-Dependent Viscosity (TDV) in a Saturated Porous Channel
}

\author{
Wissam Sadiq Khudair ${ }^{1}$, Hasan Hadi Dwail ${ }^{1}$, Hayder Kadim Mohammed ${ }^{2}$ \\ 1,2 Directorate of Education Babylon, Ministry of Education, Iraq \\ ${ }^{3}$ Directorate of Education Qadisiyah, Ministry of Education, Iraq
}

\begin{abstract}
In this research, we studied the impact of Magnetohydrodynamic (MHD) on Jeffrey fluid with porous channel saturated with temperature-dependent viscosity (TDV). It is obtained on the movement of fluid flow equations by using the method of perturbation technique in terms of number Weissenberg $(W e \ll 1)$ to get clear formulas for the field of velocity. All the solutions of physical parameters of the Reynolds number(Re), Magnetic parameter(M), Darcy parameter (Da), Peclet number $(\mathrm{Pr})$ and are discussed under the different values, as shown in the plots.
\end{abstract}

Keywords: Jeffrey fluid; Porous channel; Magnetohydrodynamic (MHD); Perturbation Technique.

\section{التدفق التذبذبي MHD لمائع جفري مع اللزوجة المعتمدة على درجة الحرارة (TDV) في قناة مسامية مشبعة

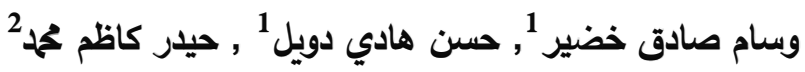 \\ مديرية تربية بابل , وزارة التربية , العراق. \\ $2{ }^{2}$ مديرية تربية القادسية , وزارة التربية , العراق.}

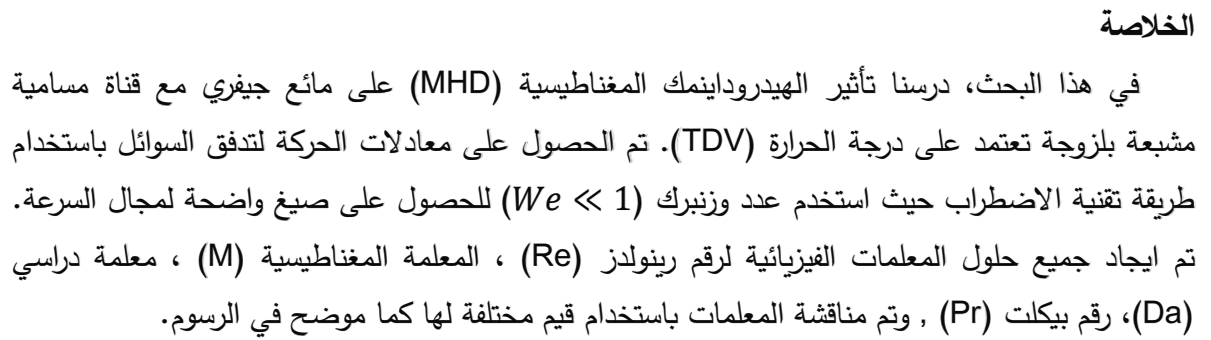

\section{Introduction}

In many biological streams and engineering requests, with arterial blood flow, Magnetohydrodynamic generators, geothermal liveliness withdrawal, petroleum engineering, atomic reactors and much more, -MHD- flux investigation of viscous liquid in a permeable channel that is full with insignificant permeable media is insignificant. The rheological fluid in the ducts is of fundamental importance for particular natural and scientific presentations of transit flux (or oscillation).

For example, the semi-intermittent flow inside the heart background container been represented by pressure (regularity component) and flow frequency beats. A lot of infections accompanying with 
the vascular method are due to a trouble in the native flow of blood vessels. In many generalized applications such as photogravure from inkjet imprinters, a quick switch is necessary between "nonflow" and "flow" for a non-Newtonian fluid.

Nadeem et al. [1] premeditated the impact of Jeffrey fluid with pressure-dependent viscosity (PDV) and there is other interesting articles studied that the effect of -PDV- in different fluids, including (Carreau fluid [2] and Williamson fluid [3]). Ajibade [4] obtainable that influence of suction /injection- on (MHD).

There are few authors extended the investigation to the impact of temperature-dependent viscosity Falade et al. [5], and Abbas et al. [6] on oscillatory flow of Casson fluid with porous channel. There are same researchers extended the problem by presenting -injection/suction- [7] and time-dependent boundary conditions (TDB) [8].

This study aims to employ a chain of perturbation method to fix the issue of an elevated medium with variable viscosity for the impact magneto hydrodynamics of temperature-dependent viscosity on Jeffrey fluid in a saturated porous channel.

\section{Preparation of the problem}

Consider the incompressible unstable flow for Magnetohydrodynamic of Jeffrey fluid with TDV - in a saturated permeable channel with variable viscosity and at height $(L)$, see (Fig.1). We choose the Cartesian coordinates system $(x, y)$ is velocity vector in which $(u)$ is the ( $x$-component) of velocity and $(y)$ is perpendicular to ( $x$-axis).

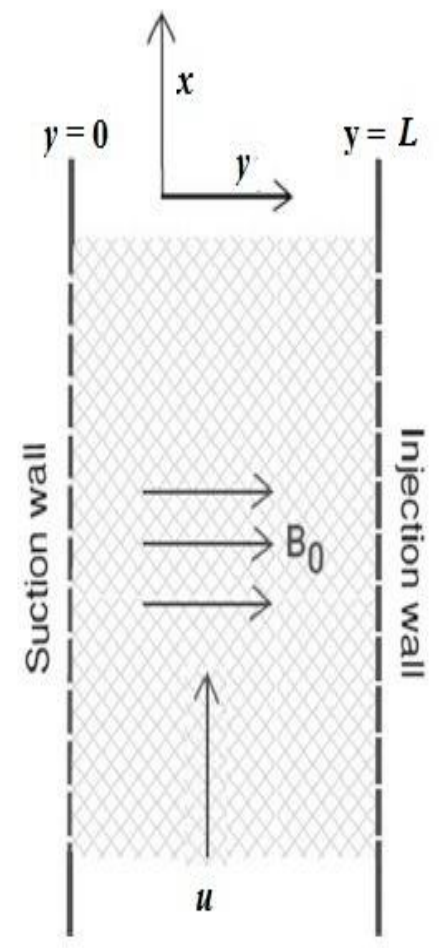

Figure 1-Construction of the Problem

The Jeffrey fluid model, the constitutive equation below [9]:

$$
\bar{\tau}=\frac{\mu(T)}{1+\lambda_{1}}\left(\dot{\gamma}+\ddot{\gamma} \lambda_{2}\right)
$$

where $\mu(T)$ is temperature-dependent viscosity, $\dot{\gamma}$ is the shear rate, $\lambda_{1}$ is the ratio of relaxation to retardation time and $\lambda_{2}$ is the retardation time.

The velocity field and the heat field of the present problem are:

$$
V=\left[u(y, t),-v_{0}, 0\right], T=T(y, t)
$$

The Jeffrey fluid model equations for flow with -TDV- are given by:

$$
\rho \frac{\partial \bar{u}}{\partial \bar{t}}-\rho v_{0} \frac{\partial \bar{u}}{\partial \bar{y}}=-\frac{\partial \bar{p}}{\partial \bar{x}}+\frac{\mu(T)}{1+\lambda_{1}} \frac{\partial^{2} \bar{u}}{\partial \bar{y}^{2}}+\rho g \beta\left(T-T_{0}\right)-\sigma B_{0}^{2} \bar{u}-\frac{\mu(T)}{\rho K} \bar{u}
$$




$$
\rho \frac{\partial T}{\partial \bar{t}}-\frac{k}{C_{p}} \frac{\partial^{2} T}{\partial \bar{y}^{2}}-v_{0} \frac{\partial T}{\partial \bar{y}}=\frac{4 b^{2}}{C_{p}}\left(T-T_{0}\right)
$$

with associated boundary conditions

$$
\left.\begin{array}{l}
\bar{u}=\frac{\sqrt{K}}{\alpha_{s}} \frac{\partial \bar{u}}{\partial \bar{y}}, T=T_{0} \text { at } \bar{y}=0 \\
\bar{u}=0, \quad T=T_{1} \text { at } \bar{y}=L
\end{array}\right\}
$$

In which $\bar{u}$ is the axial velocity, $g$ is an accelerating due to importance, $k$ is a penetrability, $\rho$ is a fluid density, $c_{p}$ is a specific heat at constant pressure, $\beta$ is a measurement of volume intensification due to heat, $q$ is a radioactive heat flux, $\sigma$ is a conductivity of the fluid, $B_{0}$ is a attractive field and $K$ is a thermal conductivity.

Non-dimensional parameters are given by (Wissam et al., 2019) [10]:

$$
\left.\begin{array}{c}
u=\frac{\bar{u} \rho L}{\mu_{0}}, x=\frac{\bar{x}}{L}, y=\frac{\bar{y}}{L}, \theta=\frac{T-T_{0}}{T_{1}-T_{0}}, t=\frac{\bar{t} V}{L}, p=\frac{\bar{p} h}{\mu_{0} V}, M^{2}=\frac{\sigma B_{0}^{2} h^{2}}{\mu_{0}} \\
R e=\frac{\rho L V}{\mu_{0}}, P r=\frac{\rho L V c_{p}}{K}, N^{2}=\frac{4 b^{2} L^{2}}{K} \mu(T)=\mu_{0} \mu(\theta) \\
G r=\frac{\rho g \beta L^{2}\left(T-T_{0}\right)}{\mu_{0} u}, S=\frac{v_{0} L \mu_{0}}{\rho}, D a=\frac{k}{L^{2}}, \eta=\frac{\sqrt{K}}{\alpha_{s} L^{2}}, \dot{\gamma}=\frac{L}{u} \overline{\dot{\gamma}},
\end{array}\right\}
$$

where $U$ is the mean flow velocity, $\left(\propto_{s}\right)$ stagnation speed, $(k)$ Porous permeability, $(\eta)$ Slip parameter, Grashof number $(G r)$, radiation parameter $(N)$, and $\left(v_{0}\right)$ Constant horizontal velocity, . Below the above expectations and with the assistance of Eqs. (1-6):

$$
\begin{aligned}
& \operatorname{Re} \frac{\partial u}{\partial t}-S \frac{\partial u}{\partial y}=-\frac{d p}{d x}+\frac{\mu(\theta)}{1+\lambda_{1}} \frac{\partial^{2} u}{\partial y^{2}}-\left(M^{2}+\frac{\mu(\theta)}{D a}\right) u+G r \theta \\
& \frac{\partial \theta}{\partial t}-S \frac{\partial \theta}{\partial y}=\frac{1}{P r} \frac{\partial^{2} \theta}{\partial y^{2}}+N^{2} \theta
\end{aligned}
$$

and Eq. (5) becomes

$$
\left.\begin{array}{l}
u=\eta \frac{\partial y}{\partial y}, \theta=0, \text { on } y=0 \\
u=0, \theta=1, \text { on } y=1
\end{array}\right\}
$$

To solve the temperature equation (8), let:

$$
\theta(y, t)=\theta_{f}(y) e^{i \omega t}
$$

Substituting the Eq. (10) into the Eq. (8), we have:

$$
\frac{\partial^{2} \theta_{f}}{\partial y^{2}}+S \times \operatorname{Pr} \frac{\partial \theta_{f}}{\partial y}+\left(N^{2}-i \omega\right) \operatorname{Pr} \times \theta_{f}=0
$$

The exact solution of Eq. (11) and Eq. (9) is given by:

$\theta_{f}(y)=\frac{1}{-1+e^{\sqrt{A^{2}-4 B}}}\left(e^{\frac{1}{2}\left(-A-\sqrt{A^{2}-4 B}\right) y}\left(-e^{\frac{A}{2}+\frac{1}{2} \sqrt{A^{2}-4 B}}\right)+e^{\frac{1}{2}\left(-A+\sqrt{A^{2}-4 B}\right) y}\left(e^{\frac{A}{2}+\frac{1}{2} \sqrt{A^{2}-4 B}}\right)\right)$,

where $A=S \times P r, B=\left(N^{2}-i \omega\right) \times P e$. Therefore

$$
\theta(y, t)=\frac{1}{-1+e^{\sqrt{A^{2}-4 B}}}\left(e^{\frac{1}{2}\left(-A-\sqrt{A^{2}-4 B}\right) y}\left(-e^{\frac{A}{2}+\frac{1}{2} \sqrt{A^{2}-4 B}}\right)+e^{\frac{1}{2}\left(-A+\sqrt{A^{2}-4 B}\right) y}\left(e^{\frac{A}{2}+\frac{1}{2} \sqrt{A^{2}-4 B}}\right)\right) e^{i \omega t}
$$

\section{Solution of the problem}

To resolve Eq. (7), with the boundary conditions (9), let:

$$
\begin{aligned}
& -\frac{d p}{\partial x}=\lambda e^{i \omega t} \\
& u(y, t)=u_{f}(y) e^{i \omega t}
\end{aligned}
$$

By substituting equations (10), (13), and (14) into Eq.(7), It follows that:

$$
\operatorname{Reiw} \frac{\partial u_{f}}{\partial t}-S \frac{\partial u_{f}}{\partial y}=\lambda+\frac{\mu(\theta)}{1+\lambda_{1}} \frac{\partial^{2} u_{f}}{\partial y^{2}}-\left(M^{2}+\frac{\mu(\theta)}{D a}\right) u_{f}+\operatorname{Gr} \theta_{f}
$$

Reynold's perfect and distinction of viscosity with heat is distinct by [11]: $\mu(\theta)=e^{-\epsilon \theta}$

by Maclaurin series:

$$
\mu(\theta)=1-\epsilon \theta, \epsilon<<1
$$

By substituting Eq. (17) into Eq. (7), have got:

$\operatorname{Reiw} \frac{\partial u_{f}}{\partial t}-S \frac{\partial u_{f}}{\partial y}=\lambda+\frac{(1-\epsilon \theta)}{1+\lambda_{1}} \frac{\partial^{2} u_{f}}{\partial y^{2}}-\left(M^{2}+\frac{(1-\epsilon \theta)}{D a}\right) u_{f}+G r \theta_{f}$

We use the -Perturbation method- to solve the equation (18), as follows [12]:

$$
u_{f}=u_{00}+W e u_{01}+\mathrm{O}\left(W e^{2}\right)
$$


By substituting Eq. (19) into Eq. (18), which equalizes the powers of $(W e)$, we obtain:

i. Zeros-order system $\left(W e^{0}\right)$

$$
\begin{aligned}
& \quad \frac{\partial^{2} u_{00}}{\partial y^{2}}-S \frac{\partial u_{09}}{\partial y}-\left(1+\lambda_{1}\right)\left(M^{2}+i w R e+\frac{1}{D a}\right) u_{00}=-\left(1+\lambda_{1}\right)\left(\lambda+G r \theta_{f}\right) \\
& u_{00}=\eta \frac{\partial u_{00}}{\partial y} \text { on } y=0 \text { and } u_{00}=0 \text { on } y=1 \\
& \text { ii. First-order system }\left(\boldsymbol{W} \boldsymbol{e}^{\mathbf{1}}\right) \\
& \quad \frac{\partial^{2} u_{01}}{\partial y^{2}}-S \frac{\partial u_{01}}{\partial y}-\left(1+\lambda_{1}\right)\left(M^{2}+i w R e+\frac{1}{D a}\right) u_{01}=\left(\frac{\partial^{2} u_{00}}{\partial y^{2}}-\frac{\left(1+\lambda_{1}\right)}{D a} u_{00}\right) \theta_{f} \\
& u_{01}=\eta \frac{\partial u_{01}}{\partial y} \text { on } y=0 \text { and } u_{01}=0 \text { on } y=1
\end{aligned}
$$

\section{Results and discussion}

In this part of the paper, magneto hydrodynamics oscillatory flow problematic of Jeffrey fluid with -TDV- in a saturated permeable channel is discoursed. The perturbation method is comprised in the direction of solving the motion equations, while the meticulous solution of temperature equation is attained. The numerical calculations were have been performed using (Mathematical ver.11) using with the set of values: $\operatorname{Re}=1, \omega=\pi, \operatorname{Pr}=1, S=1, M=1, N=1, G r=1, D a=0.8, \lambda=1, W e=$ $0.05, \lambda_{1}=0.3, \epsilon=0.02, \eta=0.1, t=0.5$.

From Figure-2, the cumulative frequency of the oscillation $\omega, \theta$ during the canal decreases is clarified. The significances payable to variation in $S$ on $\theta$ are shown in Figure-.3. From this figure, it is initially distinguished that in the absenteeism absence of (injection/suction), the fluid temperature is linearly dispersed during the channel. Conversely, the temperature of the fluid increases in the channel by increasing the injection on the heated bowl.

The linearity inspected at $\mathrm{S}=0$ provides an approach to concave circulation. By increasing $S$, the concavity is a important due to the pathway of the flow of temperature which is absorbed each time from the animated superficial to the cold superficial. From Figure-4, it is distinguished that $\theta$ increases by increasing the values of $N$ because the temperature is communicated from the animated wall near the fluid.

Figure-5 displays that velocity distribution increases, with increasing the thoughts $D a$. Figure-6 illustrates the effect of $G r$ on the velocity field. The figure demonstrates that after increasing $G r$ the velocity field $u$ increased. Figure-7 illustrations the effects of the parameter of $\lambda$. By increasing $\lambda$, velocity distribution $u$ is illustrates. Figure- 8 shows that the velocity distribution $u$ is increased with increasing $\lambda_{1}$. Figure- 9 shows the influence $M$ on the velocity field function $u$. By increasing $M$ the velocity distribution is decreased.

Figure-10 illustrates that by increasing $N$ velocity distribution $u$ is increased. Figure-11 displays that increasing $\operatorname{Pr}$, leads to an increase in velocity distribution $u$. Figure-12 it is displays that velocity distribution $u$ is decreased with increasing $R e$. Figure-13 Shows that increasing $S$ results in an increase in the velocity field is increased. Figure-14 Shows that velocity $\theta$ is decreased with increasing $\omega$.

\section{Conclusion}

The oscillatory magneto hydrodynamics flow problem of Jeffrey fluid with -TDV- in a saturated porous channel is discussed. It is established that the velocity field and temperature, examined by exhausting the perturbation method are satisfactory to resolve the problem. Different sets of values have been employed to tackle the problem. We conclude that, by increasing the $\lambda_{1}, \lambda, D a, \operatorname{Pr}, \operatorname{Gr}, S, N$ and $\operatorname{Pr}$, the velocity distribution is increased. While increasing the $M, \omega$ and $R e$ leads to a decrease in the velocity field. It is also shown that by increasing $N$ and $S$ causes an increase in the temperature but, however, increasing $\omega$ leads to a decrease in the temperature $\theta$. 


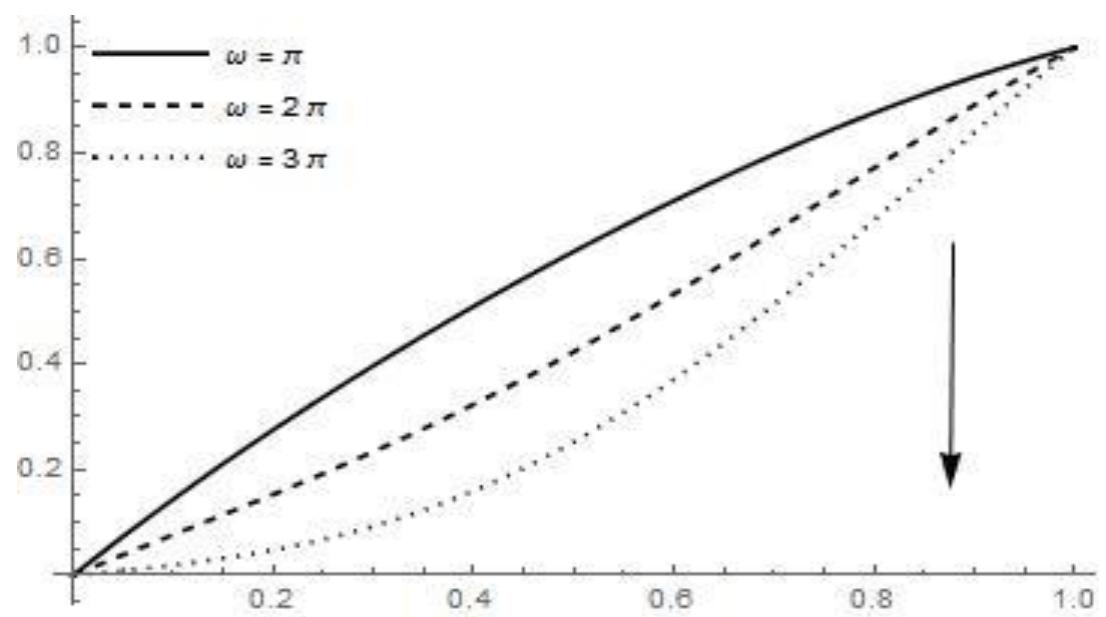

Figure 2-Impact of $\omega$ on Temperature field.

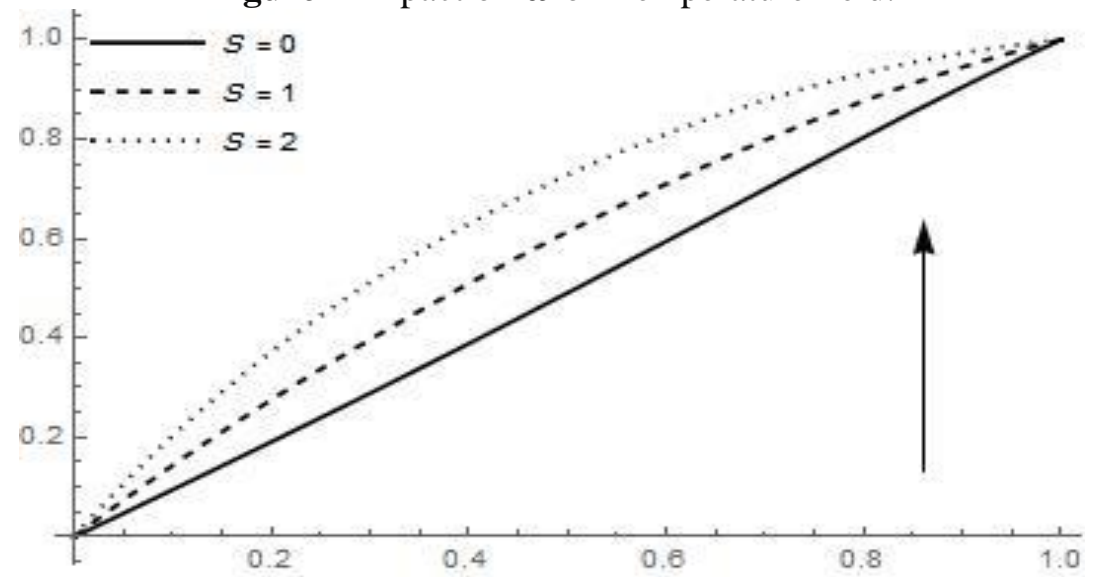

Figure 3-Impact of $S$ on Temperature field.

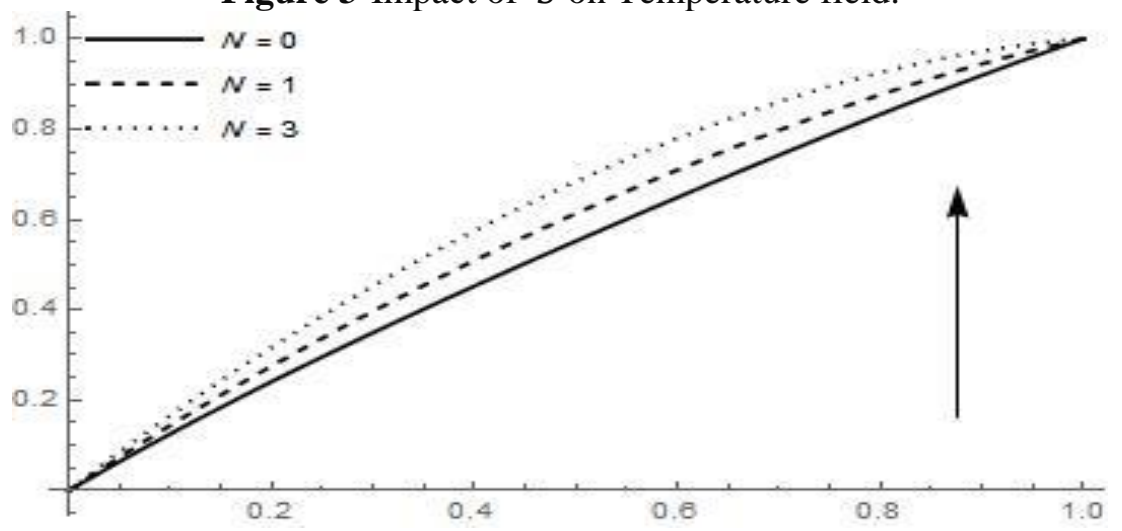

Figure 4-Impact of $N$ on Temperature field.

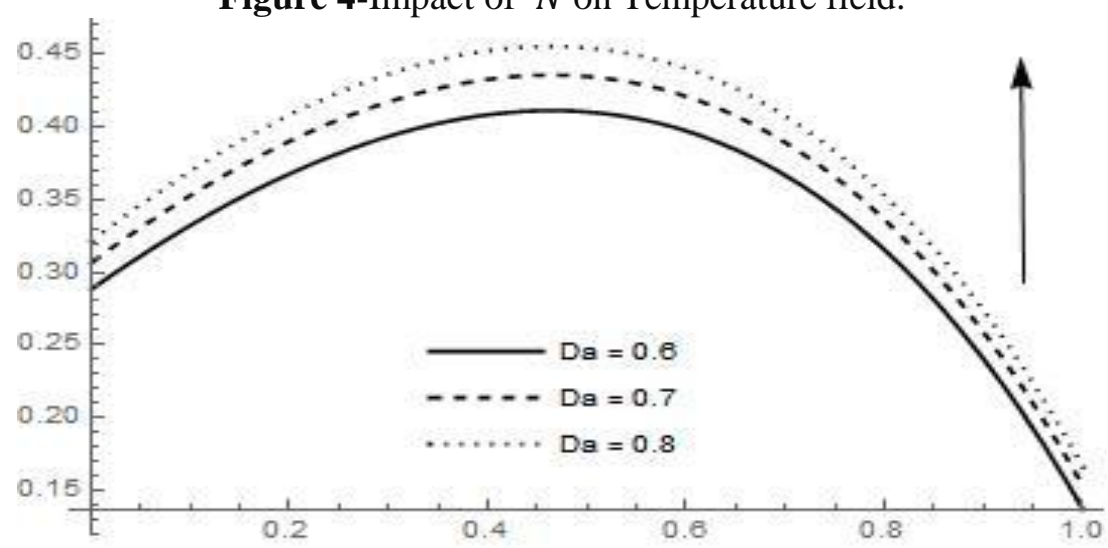

Figure 5-Impact of $\mathrm{Da}$ on Velocity distribution. 


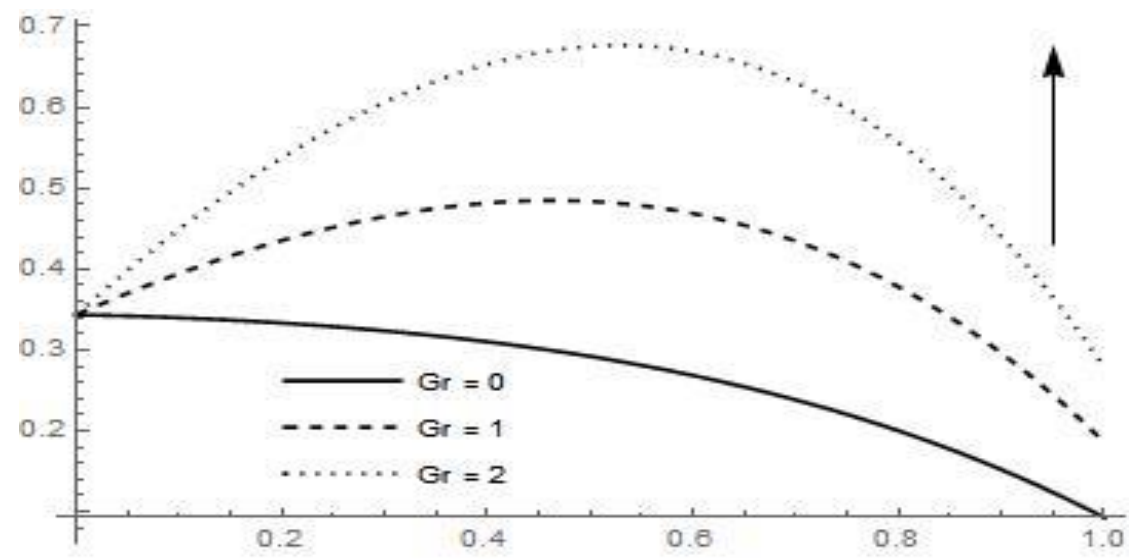

Figure 6-Impact of $\mathrm{Gr}$ on Velocity distribution.

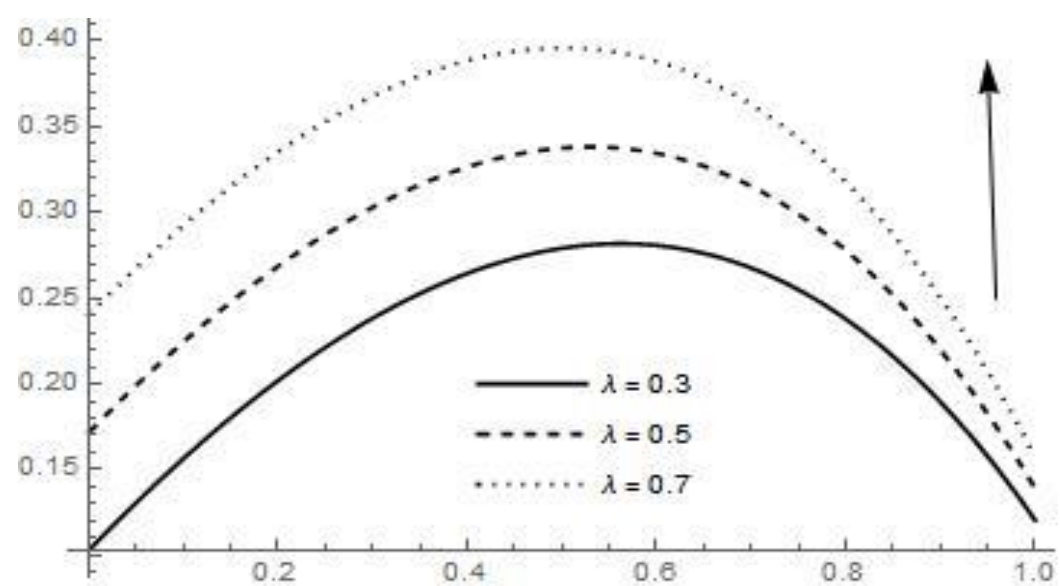

Figure 7-Impact of $\lambda$ on Velocity distribution.

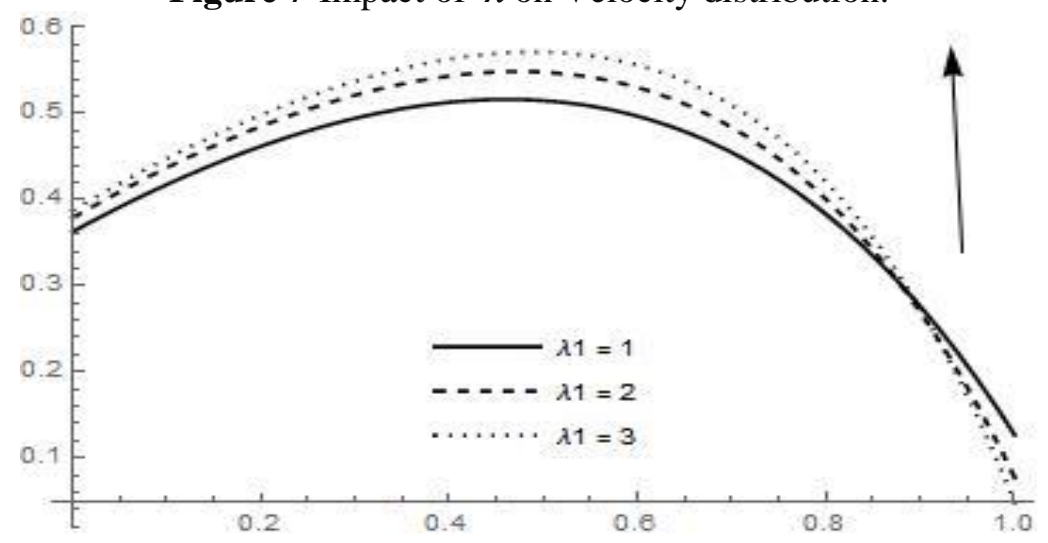

Figure 8-Impact of $\lambda_{1}$ on Velocity distribution.

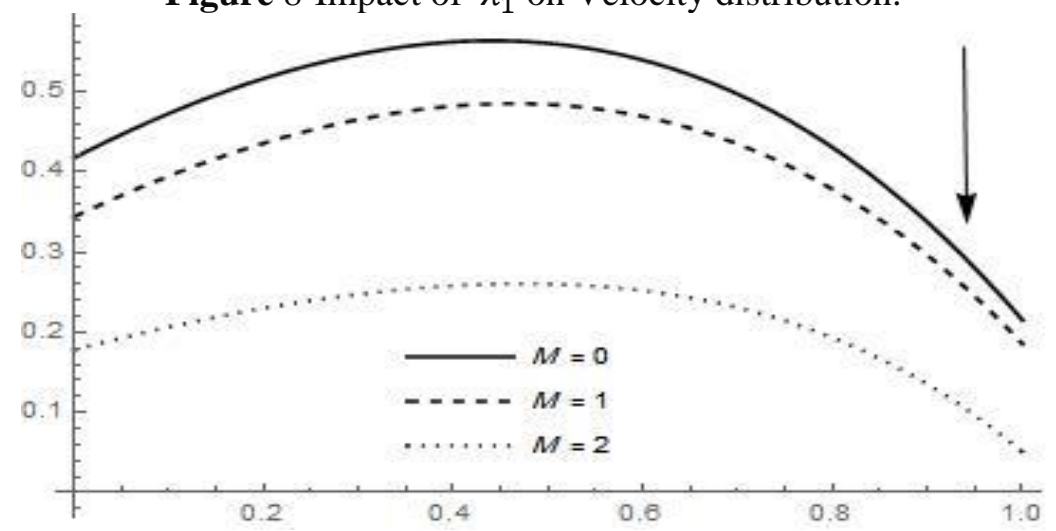

Figure 9-Impact of $M$ on Velocity distribution. 


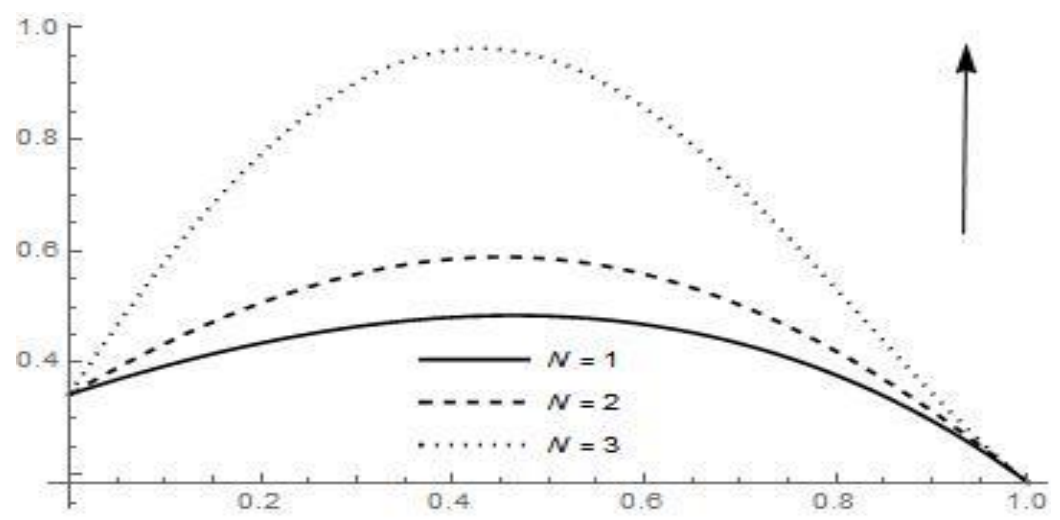

Figure 10-Impact of $N$ on Velocity distribution.

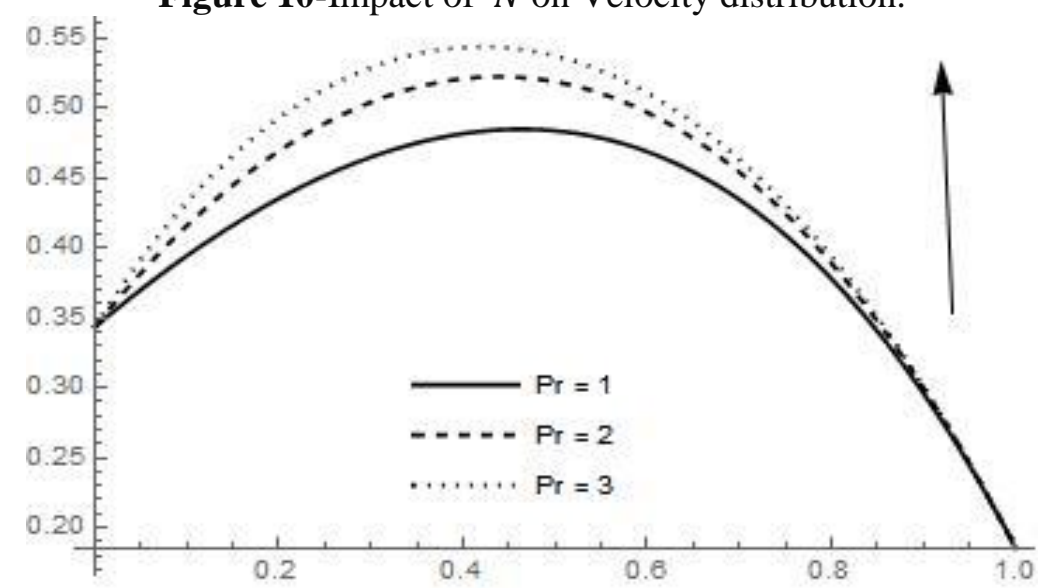

Figure 11-Impact of $\operatorname{Pr}$ on Velocity distribution.

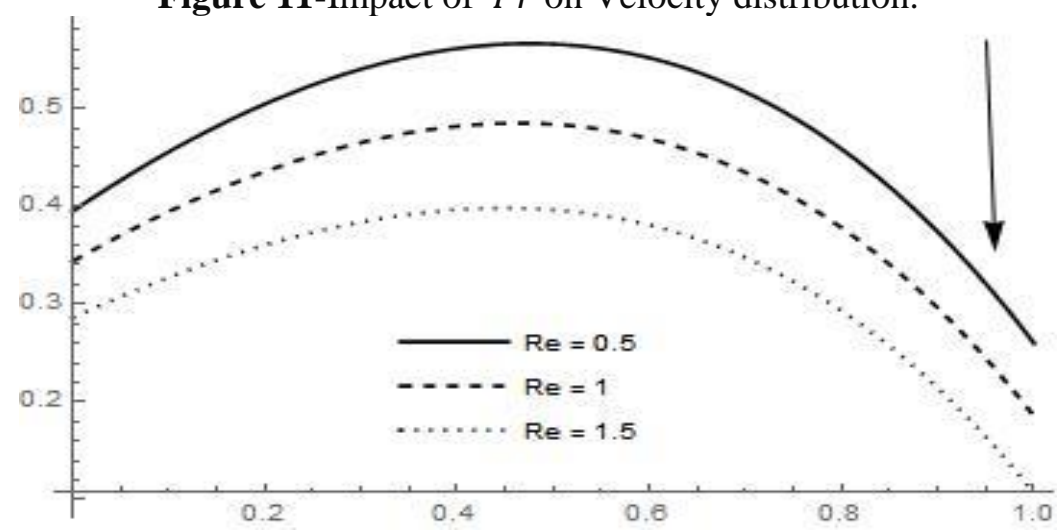

Figure 12-Impact of $R e$ on Velocity distribution.

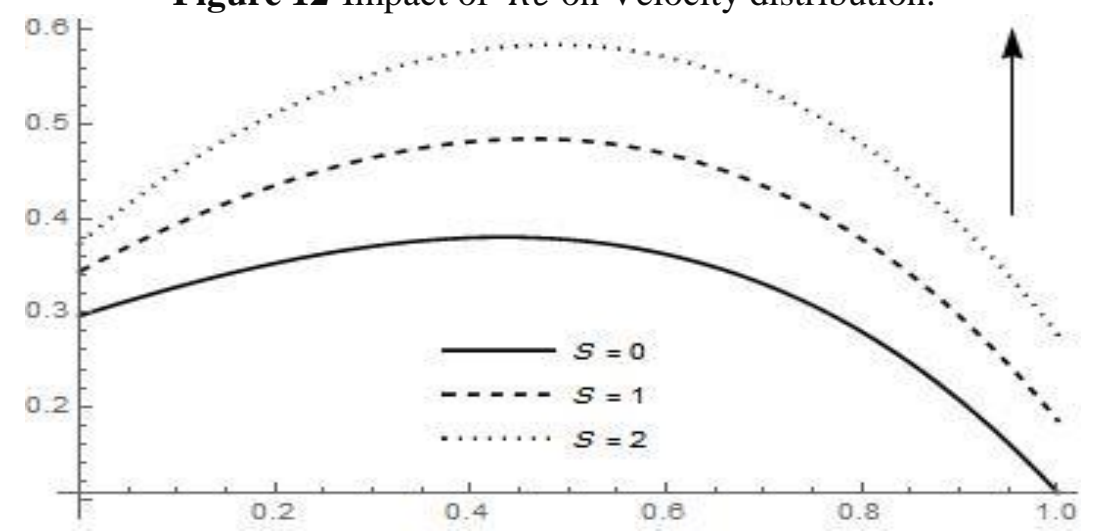

Figure 13-Impact of $S$ on Velocity distribution. 


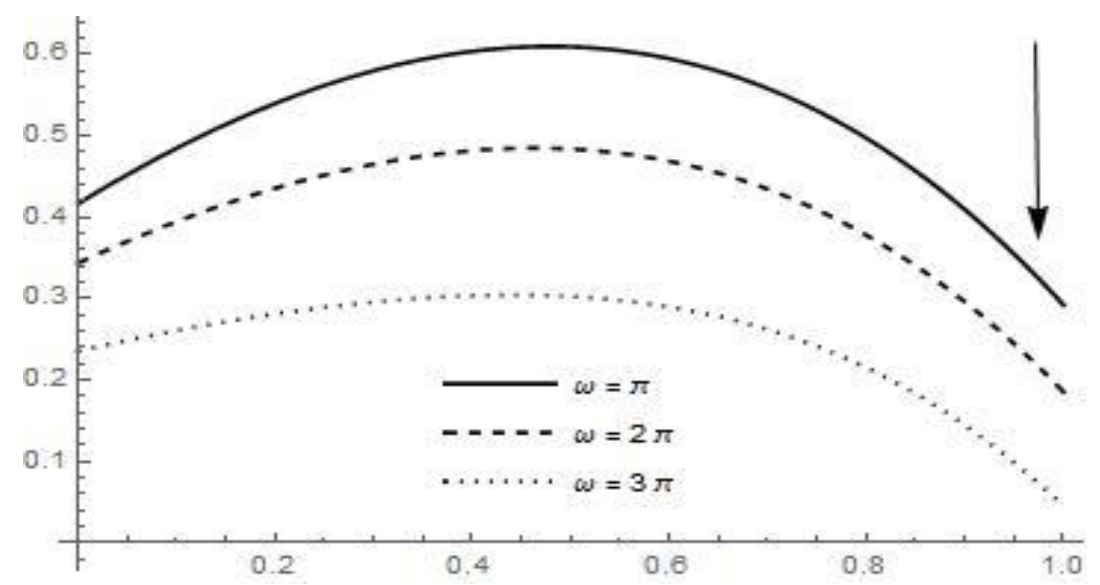

Figure 14 Impact of $\omega$ on Velocity distribution.

\section{References}

1. Malik M.Y., Zehra I. and Nadeem S. 2012. Numerical treatment of Jeffrey fluid with pressuredependent viscosity, Int. J. Numer. Meth. Fluids. 68: 196-209.

2. Malik M.Y., Zehra I., Nadeem S. 2014. Flows of Carreau fluid with pressure dependent viscosity in a variable porous medium: Application of polymer melt. Alexandria Eng. J. 53: 427-35.

3. Malik M.Y., Zehra I., Nadeem S. 2015. Numerical solutions of Williamson fluid with pressure dependent viscosity, Results in Physics, 5: 20-25.

4. Jha B.K., Ajibade A.O. 2012. Effect of viscous dissipation on natural convection flow between vertical parallel plates with time periodic boundary conditions. Commun. Nonlinear Sci. Numer. Simul. 17: 1576-1587.

5. Falade J.A., Ukaegbu J.C., Egere A.C., Adesanya S.O. 2017. MHD oscillatory flow through a porous channel saturated with porous medium. Alexandria Eng. J. 56: 147-152.

6. Abbas Z., Rafiq S., Sheikh M., Aly S. 2020. Oscillatory Darcy Flow of Non-Newtonian Casson Fluid with Temperature-Dependent Viscosity in a Porous Channel, Arabian Journal for Sci. and Eng.

7. Ellahi R. 2013. The effects of MHD and temperature dependent viscosity on the flow of nonNewtonian nanofluid in a pipe: analytical solutions. Appl Math Model, 37: 1451-7.

8. Jha B.K., Ajibade A.O. 2012. Effect of viscous dissipation on natural convection flow between vertical parallel plates with time periodic boundary conditions. Commun. Nonlinear Sci. Numer. Simul. 17: 1576-1587.

9. Bhatti M.M., Abbas A. 2016. Simultaneous effects of slip and MHD on peristaltic blood flow of Jeffrey fluid model through a porous medium. Alexandria Eng. J. 55: 10-23.

10. Hayder K.M.,Wissam S. K., Raheem J. M. and Qassim A. S. 2019. Influence of Magnetohydrodynamics Oscillatory Flow for Carreau Fluid Through Regularly Channel With Varying Temperature. Journal of Al-Qadisiyah for Computer Science and Mathematics. 11(4): 13-22.

11. Wissam S.K. and Dheia G.S. 2018. Influence of Heat Transfer on MHD Oscillatory Flow for Williamson Fluid with Variable Viscosity through a Porous Medium. Int. J. of Fluid Mec. \& Thermal Sci. 4: 11-17.

12. Romero, L. A. 2013. Perturbation theory for polynomials. Lecture Notes, University of New Mexico. 